\title{
MULTIPLE ECH LAUNCHER CONTROL SYSTEM
}

\author{
by \\ M.T. GREEN, D. PONCE, H.J. GRUNLOH, R.A. ELLIS, \\ W.H. GROSNICKLE, and R.L. HUMPHREY
}




\section{DISCLAIMER}

This report was prepared as an account of work sponsored by an agency of the United States Government. Neither the United States Government nor any agency thereof, nor any of their employees, makes any warranty, express or implied, or assumes any legal liability or responsibility for the accuracy, completeness, or usefulness of any information, apparatus, product, or process disclosed, or represents that its use would not infringe privately owned rights. Reference herein to any specific commercial product, process, or service by trade name, trademark, manufacturer, or otherwise, does not necessarily constitute or imply its endorsement, recommendation, or favoring by the United States Government or any agency thereof. The views and opinions of authors expressed herein do not necessarily state or reflect those of the United States Government or any agency thereof. 


\title{
MULTIPLE ECH LAUNCHER CONTROL SYSTEM
}

\author{
by \\ M.T. GREEN, D. PONCE, H.J. GRUNLOH, R.A. ELLIS, * \\ W.H. GROSNICKLE, and R.L. HUMPHREY*
}

*Princeton Plasma Physics Laboratory

This is a preprint of a paper presented at the 20th IEEE/NPSS Symposium on Fusion Engineering, San Diego, California, October 14-17, 2003 and to be published in Fusion Science and Technology.

\author{
Work supported by \\ the U.S. Department of Energy \\ under Contract Nos. DE-AC03-99ER54463 \\ and DE-AC02-76CH03073
}




\title{
Multiple ECH Launcher Control System
}

\author{
M.T. Green, D. Ponce, H.J. Grunloh, R.A. Ellis ${ }^{\dagger}{ }^{\dagger}$ W.H. Grosnickle, ${ }^{\dagger}$ R.L. Humphrey ${ }^{\dagger}$ \\ General Atomics, PO Box 85608, San Diego, California 92186-5608 \\ †Princeton Plasma Physics Laboratory, Princeton, New Jersey
}

\begin{abstract}
The addition of new, high power gyrotrons to the heating and current drive arsenal at DIII-D, required a system upgrade for control of fully steerable ECH Launchers. Each launcher contains two pointing mirrors with two degrees of mechanical freedom. The two flavors of motion are called facet and tilt. Therefore up to four channels of motion per launcher need to be controlled. The system utilizes absolute encoders to indicate mirror position and therefore direction of the microwave beam. The launcher movement is primarily controlled by PLC, but future iterations of design, may require this control to be accomplished by a CPU on fast bus such as Compact PCI. This will be necessary to accomplish real time position control. Safety of equipment and personnel is of primary importance when controlling a system of moving parts. Therefore multiple interlocks and fault status enunciators have been implemented. This paper addresses the design of a Multiple ECH Launcher Control System, and characterizes the flexibility needed to upgrade to a real time position control system in the future.
\end{abstract}

\section{INTRODDUCTION}

The addition of new, high power gyrotrons to the Heating and Current Drive arsenal at DIII-D, required a system upgrade in order to control multiple fully-steerable ECH Launchers.

\section{LAUNCHERS}

There are now six functional gyrotrons at DIII-D, requiring three launchers with two mirrors each to control the angles at which microwaves enter the tokamak. Earlier iterations of the ECH launcher included mirrors with only manual positioning. This meant that changing the angle of microwave direction throughout the day would require numerous access runs into the experimental hall, thus slowing down the shot cycle. As new applications of ECH heating and current drive came forth, it became clear that the ability to change the beam position between plasma shots would enhance flexibility and enrich experimental creativity. From this, the Princeton P-99 (Fig. 1) launcher was born. The P-99 launcher is fully capable of steering two independent microwave beams. It can move them toroidally independently and poloidally in a joint motion via a cradle mechanism. The mirrors roughly follow a Cartesian motion. However, the newer Princeton P01 (Fig. 2) and P02 launchers operate quite differently as far as their mechanical steering and coupling are concerned. Instead of moving mirrors in the toroidal-poloidal directions of the Cartesian coordinate system, it is designed to move in more of a Spherical coordinate system. The two flavors of motion are called facet and tilt. The motions can be described with a bicycle analogy. If one were sitting on a bicycle, looking straight down at the front wheel, and holding the handlebars, then rotating the handlebars to steer the bike would be the facet motion. Spinning the front wheel would be the tilt motion.

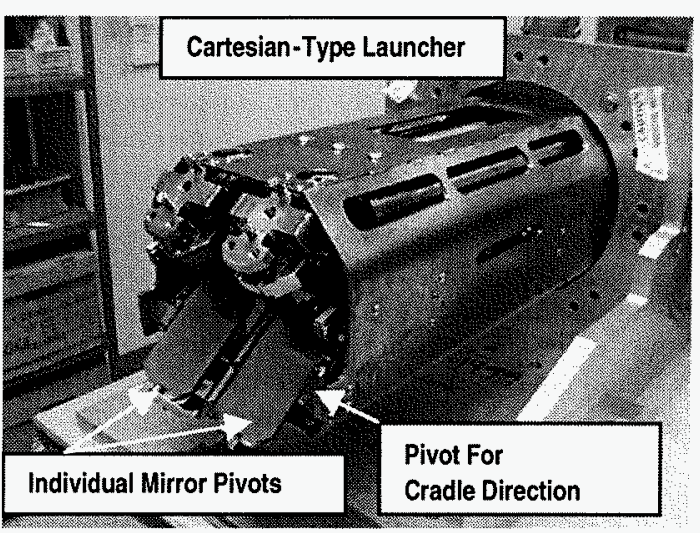

Fig. 1. P1999 launcher style.

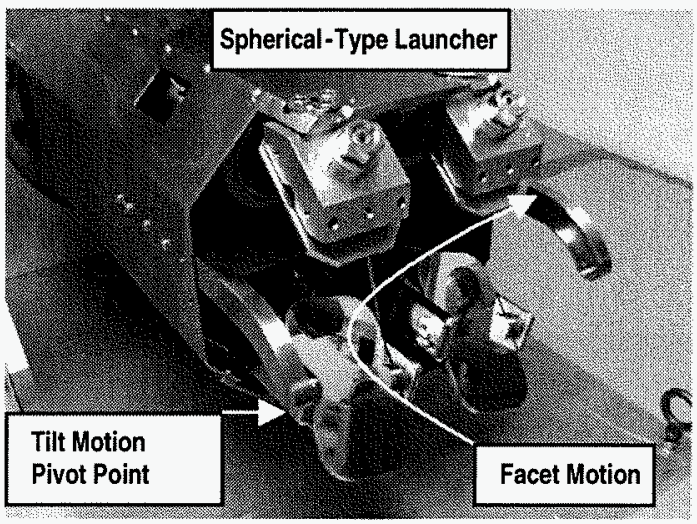

Fig. 2. P01 \& P02 launcher style.

\section{SENSORS}

There are multiple sensors mounted on each launcher. There are resistive temperature detectors (RTD's) for measuring energy transference to launcher components. There are Langmuir Probes to sense the presence of ionized gas near the launcher. A camera port is available that gives a fair view of what's going on inside the launcher. However, the most important sensors, in terms of moving the launcher mirrors, are the absolute encoders and the limit switches. The absolute encoders are an important development in launcher technology. They enable every position of a launcher mirror to be represented by a unique pair of integers, one for facet and one for tilt. The encoders offer 8 bits of resolution for every revolution of the drive shaft (i.e. 256 counts). With fairly simple interface circuitry, each encoder position is represented by a 14-bit integer. For each range of motion of a mirror, there are two hard stops. For simplicity, they are called home and away. It is not good for the mechanical parts of the launcher or for the encoders to stop moving abruptly which is exactly what would happen if the launcher mechanism were allowed to run 
full speed in any direction into a hard stop. Therefore, limit switches are mounted on each mirror moving mechanism to notify the control system when motion in a given direction is no longer possible. These limits are a safe distance from the hard mechanical stops.

\section{ACTUATORS}

Given the presence of strong magnetic fields where the launchers are mounted to the tokamak, magnetic motors were ruled out for actuation. Instead, all actuation on the launchers is done using oiled air. There is a shutter mechanism on each launcher that closes over the mirrors to protect them from being coated during baking and boronization of the DIII-D vessel. This shutter is actuated by air and its position is sensed with limit switches. An important note at this point would be that all control of the launcher is given to the $\mathrm{ECH}$ group at DIII-D with the exception of the shutter. Control of the shutter is given to the main DIII-D control room. A permit to fire microwave pulses is only given to $\mathrm{ECH}$ when the shutter position status and other interlocks are appropriately satisfied. Fig. 3 illustrates the magnitude and extent of damage that can be caused by a $\sim 800 \mathrm{~kW}$ pulse of $110 \mathrm{GHz}$ microwave energy, when the shutter is not in the fully retracted position.

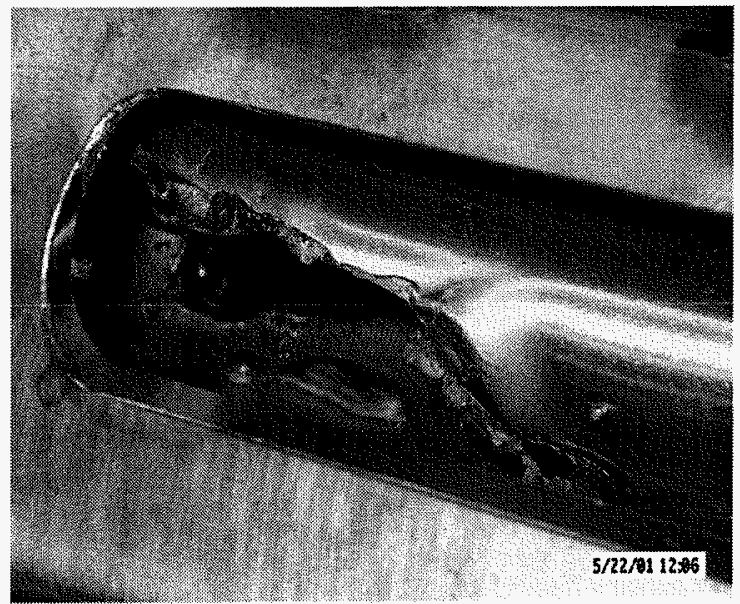

Fig. 3. Damage of waveguide due to $\mathrm{ECH}$ shot with shutter closed.

All motion of launcher mirrors is actuated using air motors. The air to the motors is controlled using solenoid controlled air manifolds. These solenoids are powered by AC power and switched by solid-state relays. Since the ability to start and stop the air motors quickly is important for a robust control system, it was decided that the air control needed to be located close to the launchers. Response time of an air motor is limited both by the length of its air hoses and the speed of sound in air.

\section{CONTROL}

The system is controlled by a Siemens PLC and programmed using Ladder Logic. However, the human interface to the system is via a Labview Virtual Instrument (Fig. 4). Labview is a graphical programming language.

The Labview VI runs on a PC in the ECH control room. It communicates to the PLC by reading and writing word vectors and status bits through the PLC serial port. Eventually, this VI
M.T. GREEN, et al

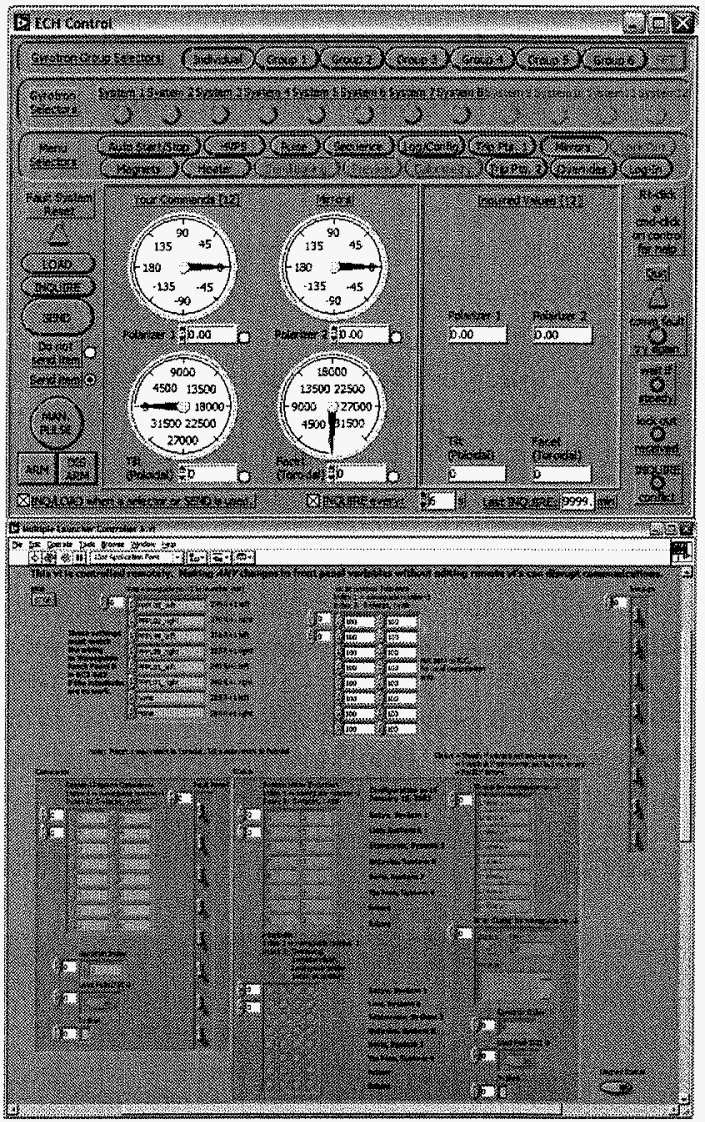

Fig. 4. Example of Labview virtual instrument panels used in the system.

may be replaced or networked to a similar interface in the main DIII-D control room.

\section{INTERLOCKS}

A permit enabling gyrotron microwave generation is interlocked to the launcher control system. There are specific circumstances that will disable a gyrotron. Although future iterations of the control system will move a launcher mirror during a plasma shot, at present we want to make sure the mirror is stationary during a shot. Therefore, a mirror-moving algorithm is implemented that checks if the mirror is in the same place it was half a second before. There has actually been a circumstance in which some type of machinery on the tokamak was vibrating the launcher in such a way as to keep the mirror from remaining stationary. In this case, the control system functioned as designed and prevented the associated gyrotron from pulsing. The mirror-moving algorithm is also useful in determining if a mirror has become stuck or is getting temporarily hung up.

There is also a "Port Boundary" interlock. If the tokamak port was rectangular and if the launcher mirror only moved up/down and left/right, a limit switch could prevent the launcher mirrors from pointing microwaves too close to the edges of the port. However, with the new P-01 and P-02 launchers we are given a slightly less than perfect scenario. The motion of the mirrors is not rectangular and the tokamak port is not rectangular or circular (Fig. 5). Therefore, a has been implemented in the PLC. For every Facet position of the launcher, there is a unique Tilt span that is allowed 
M.T. GREEN, et al.

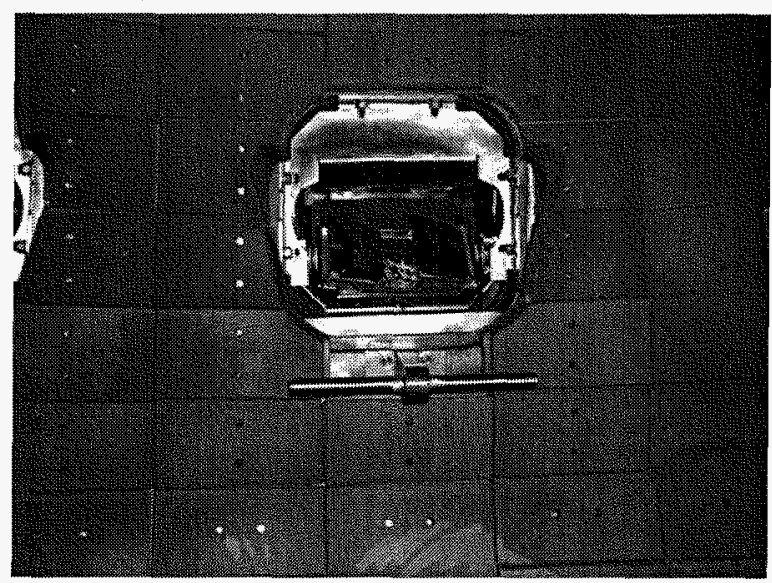

Fig. 5. An irregularly shaped tokamak portsoftware interlock.

(Fig. 6). Anything beyond this span will disable the Gyrotron from pulsing. This is actually a double interlock in that the PLC is programmed to not allow out of bounds motion and the Labview virtual instrument is programmed not to send commands to the PLC that are out of bounds.

Finally, we want the control system to announce to the operator if any motion gets stuck. Since the tokamak is under high vacuum, it is not uncommon for parts to bind up. The control system, when commanded to move, monitors the mirror position bits. If a mirror stops moving for a second, the control system shuts off motion commands and sets a fault bit. This encourages the operator to investigate why the mirror is stuck as all mirror motion is disabled until this fault bit is reset.

\section{VESSEL POTENTIAL}

DIII-D policies regarding voltage potentials, within the machine hall, state that there must be $30 \mathrm{kV}$ of isolation between the vessel and outside the machine hall. There must also be $5 \mathrm{kV}$ of isolation between the vessel potential and that of the machine hall AC power. Given that each launcher is attached to the vessel, the air control box and control cabinet wired to the launchers must also be considered to be at vessel potential, painted red and marked accordingly. The control system has parts both inside and outside the machine hall. In order to properly isolate these parts electrically, a fiber optic link is used for communication. This satisfies the $30 \mathrm{kV}$ requirement. Finally, the solenoids that trigger the air motors exist in the air control box at vessel potential. However, they are powered by machine hall $\mathrm{AC}$ power, so an isolation transformer must be used with a rating of $5 \mathrm{kV}$ or greater to isolate the vessel from machine hall power.
MULTIPLE ECH LAUNCHER CONTROL SYSTEM

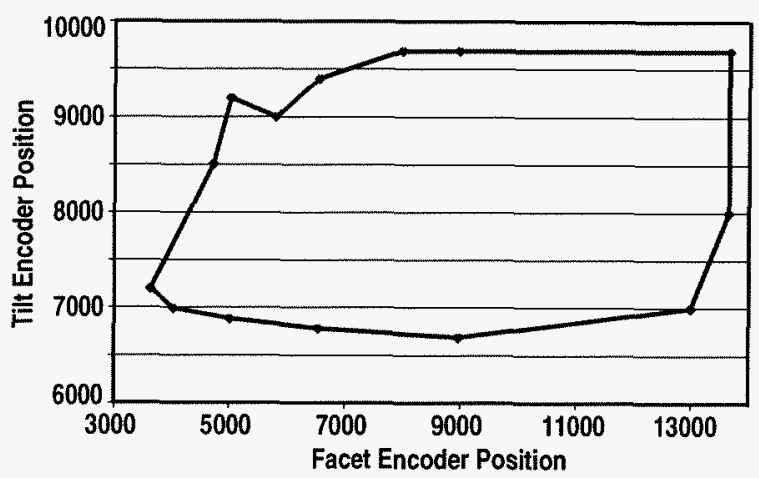

Fig. 6. Facet and tilt span lmits.

\section{THE FUTURE OF THE SYSTEM}

\section{A. Real-Time Position Control}

Eventually, there is a project goal for the system to scan between different locations in the vessel during a plasma shot. An existing system at DIII-D called the plasma control system (PCS) currently adjusts the position of the plasma during the shot to optimize the ECH. It is believed that if the PCS commanded the launcher control system, it would be able to scan the ECH in real-time to further enhance the mode suppression capabilities of ECH. Therefore, design work is currently under way to convert the existing system to one that operates via commands from the PCS, moves the mirror accordingly, and stores position vs. time in memory. Since the system is currently controlled by a PLC, and PLC's are not designed for data acquisition, the control system will probably need to be transitioned to a microprocessor-type control system using a fast bus such as PCI.

\section{B. Electromagnetic Motors}

It has been recently decided that a DC motor has the capability of a quicker response time than an air turbine style motor. The air motor runs quickly but based on the length of the air hoses, cannot start and stop with enough precision. Despite the disadvantages of using a magnetic motor in a magnetically rich environment, it has been decided that with a long enough input shaft, electromagnetic motors may be more suitable for the type of physics experiments envisioned in the near future for DIII-D. Therefore, work is taking place for a transition of the control system to handle DC motors or some other electrically driven motor system.

\section{ACKNOWLEDGMENT}

Work supported by U.S. Department of Energy Contract Nos. DE-AC03-99ER54463 and DE-AC02-76CH03073. 